DE GRUYTER

F A S C I C U L I M A T H E M A T I C I

Nr 59

C. Kongban, and P. Kumam

\title{
SOME RANDOM COUPLED BEST PROXIMITY POINTS FOR A GENERALIZED $\omega$-CYCLIC CONTRACTION IN POLISH SPACES
}

\begin{abstract}
In this paper, we will introduce the concepts of a random coupled best proximity point and then we prove the existence of random coupled best proximity points in separable metric spaces. Our results extend the previous work of Akbar et al. [1]. KEY WORDS: random fixed point, random operator, measurable mappings, coupled best proximity point, random best proximity point.
\end{abstract}

AMS Mathematics Subject Classification: 47H10, 54H25.

\section{Introduction}

A random fixed point theorem is a stochastic generalization of classical fixed point theorems. Random fixed point theorems for random contraction mappings on separable complete metric spaces were first proved by Špaček [33] and Hanš [13, 12]. Subsequently, Bharucha-Reid [6] proved the stochastic version of the well-known as Banachs and Schauders fixed point theorems and hence random fixed point theory and applications have been developed rapidly in recent years, see, e.g., Bharucha-Reid [5], Itoh [15], Choudhury [7], Beg [3], Beg and Shahzad [4], Kumam [22, 17, 18, 23], Kuman and Plubtieng [19, 21, 20], and Hussain et al. [14]. Ćirić and Lakshmikantham [8], Zhu and Xiao [35], and Khan et al. [16] proved some coupled random fixed point and coupled random coincidence point results in partially ordered complete metric spaces.

In 1969, Fan [10] has established a classical best approximation theorem and notion of a best proximity under the conditions that if $A$ is a nonempty compact convex subset of a Hausdorff locally convex topological vector space $B$ and $T: A \rightarrow B$ is a continuous mapping, then there exists an element $a \in A$ such that $d(a, T a)=d(T a, A)$. Subsequently, many researchers have 
studied the existence and convergence of best proximity point results in many ways $[9,26,24,25,27,29,32,31]$.

In 2011, Anh [2] introduced the concept of random best proximity point of a random operator. In 2012, Sintunavarat and Kumam [30] introduced the concept of a coupled best proximity point and proved the existence and uniqueness of the coupled best proximity point in metric and uniformly convex Banach spaces. In 2014, Gupta et al. [11] introduced a new class of generalized cyclic contraction mappings. Recently, Akbar et al. [1] proved some random coupled best proximity point theorems which extended the results of Sintunavarat and Kumam [30].

In this work, we introduce the concepts of a random coupled best proximity point. We also prove some new results about random best proximity points in a Polish space which extend the previous work of Akbar et al. [1].

\section{Preliminaries}

Given $A$ and $B$ be nonempty subsets of a metric space $(X, d)$, the following notations are used subsequently. We let

$$
d(A, B)=\inf \{d(x, y): x \in A, y \in B\},
$$

stands for the distance between $A$ and $B$.

Definition 1. A Banach space $X$ is said to be :

(i) strictly convex if for all $x, y \in X,\|x\|=\|y\|=1$ and $x \neq y$ imply that $\left\|\frac{x+y}{2}\right\|<1$,

(ii) uniformly convex if for each $\epsilon$ with $0<\epsilon<2$, there exists a $\delta>0$ such that $\|x\| \leq 1,\|y\| \leq 1$ and $\|x-y\| \geq \epsilon \Rightarrow\left\|\frac{x+y}{2}\right\|<1-\delta$ for all $x, y \in X$.

Definition 2 ([31]). Given $A, B$ be nonempty subsets of a metric space $(X, d)$. The odered pair $(A, B)$ is said to satisfy the property $U C$ if the following holds:

If $\left\{x_{n}\right\}$ and $\left\{z_{n}\right\}$ are sequences in $A$ and $\left\{y_{n}\right\}$ is a sequence in $B$ such that $d\left(x_{n}, y_{n}\right) \rightarrow d(A, B)$ and $d\left(z_{n}, y_{n}\right) \rightarrow d(A, B)$, then $d\left(x_{n}, z_{n}\right) \rightarrow 0$.

Definition 3 ([30]). Given $A, B$ be nonempty subsets of a metric space $(X, d)$. The ordered pair $(A, B)$ has the property $U C^{*}$ if $(A, B)$ has the property $U C$ and the following condition holds:

(i) $d\left(z_{n}, y_{n}\right) \rightarrow d(A, B)$,

(ii) for every $\epsilon>0$ there exists an $N \in \mathbb{N}$ such that

$$
d\left(x_{m}, y_{n}\right) \leq d(A, B)+\epsilon
$$

for all $m>n \geq N$, then for every $\epsilon>0$ there exists an $N_{1} \in \mathbb{N}$ such 
that

$$
d\left(x_{m}, z_{n}\right) \leq d(A, B)+\epsilon
$$

for all $m>n \geq N_{1}$.

Definition 4. Given $A, B$ be nonempty subsets of a metric space $(X, d)$. Given mapping $S: A \rightarrow B$. A point $a \in A$ is said to be a best proximity point of $S$ if

$$
d(a, S a)=d(A, B) .
$$

Definition 5. Given $A, B$ be nonempty subsets of a metric space $(X, d)$. Given mapping $S: A \times A \rightarrow B$. A point $\left(a, a^{\prime}\right) \in A \times A$ is said to be $a$ coupled best proximity point of $S$ if

$$
d\left(a, S\left(a, a^{\prime}\right)\right)=d\left(a^{\prime}, S\left(a^{\prime}, a\right)\right)=d(A, B)
$$

Definition 6 ([30]). Given $A, B$ be nonempty subsets of a metric space $(X, d)$ and let $S: A \times A \rightarrow B$ and $T: B \times B \rightarrow A$ be two maps. The ordered pair $(S, T)$ is said to be a cyclic contraction if there exists a nonnegative number $\alpha<1$ such that

$$
d\left(S\left(a, a^{\prime}\right), T\left(b, b^{\prime}\right)\right) \leq \frac{\alpha}{2}\left[d(a, b)+d\left(a^{\prime}, b^{\prime}\right)\right]+(1-\alpha) d(A, B),
$$

for all $\left(a, a^{\prime}\right) \in A \times A$ and $\left(b, b^{\prime}\right) \in B \times B$.

Definition 7 ([11]). Given $A, B$ be nonempty subsets of a metric space $(X, d)$ and let $S: A \times A \rightarrow B$ and $T: B \times B \rightarrow A$ be two maps. The ordered pair $(S, T)$ is said to be a generalized cyclic contraction if there exists a nonnegative number $l+c<1$ such that

$$
d\left(S\left(a, a^{\prime}\right), T\left(b, b^{\prime}\right)\right) \leq\left[l d(a, b)+c d\left(a^{\prime}, b^{\prime}\right)\right]+(1-(l+c)) d(A, B),
$$

for all $\left(a, a^{\prime}\right) \in A \times A$ and $\left(b, b^{\prime}\right) \in B \times B$.

Definition 8. Let $(\Omega, \Sigma)$ be a measurable space with $\Sigma$ a sigma algebra of subsets of $\Omega$ and let $(X, d)$ be a metric space. A mapping $T: \Omega \rightarrow X$ is said to be a $\Sigma$-measurable if for any open subset $U$ of $X$, one has $T^{-1}(U)=$ $\{\omega: T(\omega) \in U\} \in \Sigma$.

Definition 9. A mapping $T: \Omega \times X \rightarrow X$ is said to be a random operator if for any $x \in X, T(\cdot, x)$ is measurable.

Definition 10. A measurable mapping $\delta: \Omega \rightarrow X$ is a random fixed point of a random mapping $S: \Omega \times X \rightarrow X$ if $\delta(\omega)=S(\omega, \delta(\omega))$ for each $\omega \in \Omega$. 
Definition 11 ([2]). Given $A, B$ be two closed subsets of a complete separable metric space $X$. Given $S: \Omega \times A \rightarrow B$ be a random operator. $A$ measurable mapping $\delta: \Omega \rightarrow A$ is said to be a random best proximity point of $S$ if

$$
d(\delta(\omega), S(\omega, \delta(\omega)))=d(A, B)
$$

for each $\omega \in \Omega$.

Definition 12 ([1]). Given $A, B$ be nonempty subsets of a separable metric space $(X, d)$ and $S: \Omega \times(A \times A) \rightarrow B$ be a random operator. $A$ measurable mappings $\delta, \eta: \Omega \rightarrow A$ are called a random coupled best proximity point of $S$ if

$$
\begin{aligned}
& d(\delta(\omega), S(\omega,(\delta(\omega), \eta(\omega))))=d(A, B), \\
& d(\eta(\omega), S(\omega,(\eta(\omega), \delta(\omega))))=d(A, B),
\end{aligned}
$$

for each $\omega \in \Omega$.

\section{Main results}

In this section after giving a generalization of Definition 7, now we extend generalized cyclic contraction to random version and some new results are obtained.

Definition 13. Let $A, B$ be nonempty subsets of a separable metric space $(X, d)$ and $(\Omega, \Sigma)$ be a measurable space, $S: \Omega \times(A \times A) \rightarrow B$ and $T: \Omega \times(B \times B) \rightarrow A$ be two random operators. The ordered pair $(F, G)$ is said to be a generalized $\omega$-cyclic contraction if there exists a nonnegative number $l$ and $c$ with $l+c<1$ such that

$$
\begin{aligned}
& d\left(S\left(\omega,\left(a(\omega), a^{\prime}(\omega)\right)\right), T\left(\omega,\left(b(\omega), b^{\prime}(\omega)\right)\right)\right) \\
& \quad \leq\left[l d(a(\omega), b(\omega))+c d\left(a^{\prime}(\omega), b^{\prime}(\omega)\right)\right]+(1-(l+c)) d(A, B),
\end{aligned}
$$

for all $\left(a, a^{\prime}\right) \in A \times A$ and $\left(b, b^{\prime}\right) \in B \times B$.

Example 1. Let $X=\mathbb{R}$ with the usual metric $d(x, y)=|x-y|$. Let $\Omega=[0,1]$ and let $\sigma$ be the sigma algebra of Lebesgues measurable subset of $[0,1]$. Let $A=[6,12]$ and $B=[-12,-6]$. It easy to see that $d(A, B)=12$. Define random operators $S: \Omega \times(A \times A) \rightarrow B$ and $T: \Omega \times(B \times B) \rightarrow A$ by

$$
S\left(\omega,\left(a(\omega), a^{\prime}(\omega)\right)\right)=\frac{-3 a(\omega)-2 a^{\prime}(\omega)-6}{6},
$$

and

$$
T\left(\omega,\left(b(\omega), b^{\prime}(\omega)\right)\right)=\frac{-3 b(\omega)-2 b^{\prime}(\omega)+6}{6} .
$$


For arbitrary $\left(a(\omega), a^{\prime}(\omega)\right) \in A \times A$ and $\left(b(\omega), b^{\prime}(\omega)\right) \in B \times B$ and fixed $l=\frac{1}{2}$ and $c=\frac{1}{3}$, we get

$$
\begin{aligned}
d & \left.S\left(\omega,\left(a(\omega), a^{\prime}(\omega)\right)\right), T\left(\omega,\left(b(\omega), b^{\prime}(\omega)\right)\right)\right) \\
& =\left|\frac{-3 a(\omega)-2 a^{\prime}(\omega)-6}{6}-\frac{-3 b(\omega)-2 b^{\prime}(\omega)+6}{6}\right| \\
& \leq \frac{3|a(\omega)-b(\omega)|+2\left|a^{\prime}(\omega)-b^{\prime}(\omega)\right|}{6}+2 \\
& =\left[l d(a(\omega), b(\omega))+c d\left(a^{\prime}(\omega), b^{\prime}(\omega)\right)\right]+(1-(l+c) d(A, B) .
\end{aligned}
$$

This implies that $(S, T)$ is a generalized $\omega$-cyclic contraction with $l=\frac{1}{2}$ and $c=\frac{1}{3}$.

Theorem 1. Given $(X, d)$ be a Polish space. Let $(\Omega, \Sigma)$ be a measurable space and $A, B$ be nonempty closed subsets of $X$. Suppose that $S: \Omega \times(A \times$ $A) \rightarrow B$ and $T: \Omega \times(B \times B) \rightarrow A$ be two random operators. Define

$$
\begin{aligned}
& x_{n+1}(\omega)=S\left(\omega,\left(x_{n}(\omega), y_{n}(\omega)\right)\right), \\
& y_{n+1}(\omega)=S\left(\omega,\left(y_{n}(\omega), x_{n}(\omega)\right)\right)
\end{aligned}
$$

and

$$
\begin{aligned}
& x_{n+2}(\omega)=T\left(\omega,\left(x_{n+1}(\omega), y_{n+1}(\omega)\right)\right), \\
& y_{n+2}(\omega)=T\left(\omega,\left(y_{n+1}(\omega), x_{n+1}(\omega)\right)\right)
\end{aligned}
$$

for all $n \in \mathbb{N}$ and $\omega \in \Omega$. Let $S$ be continuous and suppose that

(i) $S(\cdot, v)$ and $T(\cdot, u)$ are measurable for all $s \in A \times A$ and $t \in B \times B$ respectively;

(ii) $(A, B)$ and $(B, A)$ have the property $U C^{*}$;

(iii) $(S, T)$ is a generalized $\omega$-cyclic contraction.

Then $S$ and $T$ have a random coupled best proximity point.

Proof. Given $\Theta=\{\zeta: \Omega \rightarrow X\}$ be a family of measurable mappings. Define $h=\Omega \times X \rightarrow \mathbb{R}^{+}$by

$$
h(\omega, x)=d(x, S(\omega, x)) .
$$

Since $x \rightarrow S(\omega, x)$ is a continuous for all $\omega \in \Omega$, it can be concluded that $h(\omega, \cdot)$ is continuous function for all $\omega \in \Omega$. Also, since $x \rightarrow S(\omega, x)$ is measurable for all $x \in X$, it can be concluded that $h(\omega, \cdot)$ is measurable for all $\omega \in \Omega$ (see [34]). So $h(\omega, x)$ is the Caratheodory function. Thus, if $\delta: \Omega \rightarrow X$ is a measurable mapping, then $\omega \rightarrow h(\omega, \delta(\omega))$ is also measurable(see[28]). Also, for each $\delta \in \Theta$, the function $\eta: \Omega \rightarrow X$ defined by $\eta(\omega)=S(\omega, \delta(\omega))$ is also measurable, that is, $\eta \in \Theta$. Now, we shall 
construct two sequences $\left\{\delta_{n}(\omega)\right\}$ and $\left\{\eta_{n}(\omega)\right\}$ of measurable mappings in $\Omega$ and will prove the theorem in three steps:

Step I. For each $n \in \mathbb{N} \cup\{0\}$ from (1) and (2), we have

$$
\begin{aligned}
d\left(\delta_{n}(\omega), \delta_{n+1}(\omega)\right)= & d\left(S\left(\omega,\left(\delta_{n}(\omega), \eta_{n}(\omega)\right)\right), T\left(\omega,\left(\delta_{n-1}(\omega), \eta_{n-1}(\omega)\right)\right)\right) \\
\leq & {\left[l d\left(\delta_{n}(\omega), \delta_{n-1}(\omega)\right)+c d\left(\eta_{n}(\omega), \eta_{n-1}(\omega)\right)\right] } \\
& +(1-(l+c)) d(A, B) .
\end{aligned}
$$

Similarly, we have

$$
\begin{aligned}
d\left(\eta_{n}(\omega), \eta_{n+1}(\omega)\right)= & d\left(S\left(\omega,\left(\eta_{n}(\omega), \delta_{n}(\omega)\right)\right), T\left(\omega,\left(\eta_{n-1}(\omega), \delta_{n-1}(\omega)\right)\right)\right) \\
\leq & {\left[l d\left(\eta_{n}(\omega), \eta_{n-1}(\omega)\right)+c d\left(\delta_{n}(\omega), \delta_{n-1}(\omega)\right)\right] } \\
& +(1-(l+c)) d(A, B) .
\end{aligned}
$$

Therefore, by letting

$$
d_{n}=d\left(\delta_{n}(\omega), \delta_{n+1}(\omega)\right)+d\left(\eta_{n}(\omega), \eta_{n+1}(\omega)\right)
$$

by adding above inequality we have

$$
d_{n} \leq(l+c) d_{n-1}+2(1-(l+c)) d(A, B) .
$$

Similarly we must show that

$$
d_{n-1} \leq(l+c) d_{n-2}+2(1-(l+c)) d(A, B) .
$$

Consequently we have

$$
d_{1} \leq(l+c) d_{n-2}+2(1-(l+c)) d(A, B) .
$$

If $d_{0}=0$ then $\left(\delta_{0}(\omega), \eta_{0}(\omega)\right)$ is a random coupled best proximity point of $S$ and $T$.

Now let $d_{0}>0$ for each $n \geq m$, we have

$$
\begin{aligned}
d\left(\delta_{n}(\omega), \delta_{m}(\omega)\right) \leq & d\left(\delta_{n}(\omega), \delta_{n-1}(\omega)\right)+d\left(\delta_{n-1}(\omega), \delta_{n-2}(\omega)\right)+\ldots \\
& +d\left(\delta_{m+1}(\omega), \delta_{m}(\omega)\right) \\
d\left(\eta_{n}(\omega), \eta_{m}(\omega)\right) \leq & d\left(\eta_{n}(\omega), \eta_{n-1}(\omega)\right)+d\left(\eta_{n-1}(\omega), \eta_{n-2}(\omega)\right)+\ldots \\
& +d\left(\eta_{m+1}(\omega), \eta_{m}(\omega)\right)
\end{aligned}
$$

which gives

$$
\begin{gathered}
d\left(\delta_{n}(\omega), \delta_{m}(\omega)\right)+d\left(\eta_{n}(\omega), \eta_{m}(\omega)\right) \leq d_{n-1}+d_{n-2} d_{n-3}+\ldots+d_{m} \\
d_{n} \leq(l+c)^{n} d_{0}+2^{n}\left(1-(l+c)^{n}\right) d(A, B) .
\end{gathered}
$$


Letting $n \rightarrow \infty$, we obtain

$$
d\left(\delta_{n}(\omega), \delta_{m}(\omega)\right)+d\left(\eta_{n}(\omega), \eta_{m}(\omega)\right) \rightarrow d(A, B)
$$

implies that

$$
d\left(\left(\delta_{n}(\omega),\left(\delta_{n+1}(\omega)\right) \rightarrow d(A, B) .\right.\right.
$$

By similar arguments, we can prove that

$$
\begin{aligned}
d\left(\left(\delta_{n+1}(\omega),\left(\delta_{n+2}(\omega)\right)\right.\right. & \rightarrow d(A, B) . \\
d\left(\left(\eta_{n}(\omega),\left(\eta_{n+1}(\omega)\right)\right.\right. & \rightarrow d(A, B) . \\
d\left(\left(\eta_{n+1}(\omega),\left(\eta_{n+2}(\omega)\right)\right.\right. & \rightarrow d(A, B) .
\end{aligned}
$$

Now, we have to show that for every $\epsilon>0$, there exists a positive integer $N_{0}$ such that for all $m>n>N_{0}$,

$$
l d\left(\delta_{m}(\omega), \delta_{n+1}(\omega)\right)+c d\left(\eta_{m}(\omega), \eta_{n+1}(\omega)\right)<d(A, B)+\epsilon .
$$

Since the pairs $(A, B)$ has the property $\mathrm{UC}$, we get

$$
d\left(\delta_{n}, \delta_{n+2}\right) \rightarrow 0, \text { as } n \rightarrow \infty .
$$

A similar argument shows that

$$
d\left(\eta_{n}, \eta_{n+2}\right) \rightarrow 0, \text { as } n \rightarrow \infty .
$$

As the pairs $(B, A)$ has the property $\mathrm{UC}$, we also have

$$
\begin{gathered}
d\left(\delta_{n+1}, \delta_{n+3}\right) \rightarrow 0 \\
d\left(\eta_{2 n+1}, \eta_{2 n+3}\right) \rightarrow 0 .
\end{gathered}
$$

Assume contrary that (7) does not hold. Then there would exists an $\epsilon^{\prime}$ such that for all $k \in \mathbb{N}$, there would be an $m_{k}>n_{k} \geq k$ satisfying

$$
l d\left(\delta_{m_{k}}(\omega), \delta_{n_{k}+1}(\omega)\right)+c d\left(\eta_{m_{k}}(\omega), \eta_{n_{k}+1}(\omega)\right) \geq d(A, B)+\epsilon,
$$

and

$$
l d\left(\delta_{m_{k}-2}(\omega), \delta_{n_{k}+1}(\omega)\right)+c d\left(\eta_{m_{k}-2}(\omega), \eta_{n_{k}+1}(\omega)\right)<d(A, B)+\epsilon .
$$

That is, we would have

$$
\begin{aligned}
d(A, B)+\epsilon^{\prime}= & l d\left(\delta_{m_{k}}(\omega), \delta_{n_{k}+1}(\omega)\right)+c d\left(\eta_{m_{k}}(\omega), \eta_{n_{k}+1}(\omega)\right) \\
\leq & l\left[d\left(\delta_{m_{k}}(\omega), \delta_{m_{k}-2}(\omega)\right)+d\left(\delta_{m_{k}-2}(\omega), \delta_{n_{k}+1}(\omega)\right)\right] \\
& +c\left[d\left(\eta_{m_{k}}(\omega), \eta_{m_{k}-2}(\omega)\right)+d\left(\eta_{m_{k}-2}(\omega), \eta_{n_{k}+1}(\omega)\right)\right] \\
< & l d\left(\delta_{m_{k}}(\omega), \delta_{m_{k}-2}(\omega)\right)+c d\left(\eta_{m_{k}}(\omega), \eta_{m_{k}-2}(\omega)\right) \\
& +d(A, B)+\epsilon^{\prime} .
\end{aligned}
$$


Letting $k \rightarrow \infty$, we obtain to see that

$$
l d\left(\delta_{m_{k}}(\omega), \delta_{n_{k}+1}(\omega)\right)+c d\left(\eta_{m_{k}}(\omega), \eta_{n_{k}+1}(\omega)\right) \rightarrow d(A, B)+\epsilon^{\prime} .
$$

By using the triangle inequality, we get

$$
\begin{aligned}
& l d\left(\delta_{m_{k}}(\omega), \delta_{n_{k}+1}(\omega)\right)+c d\left(\eta_{m_{k}}(\omega), \eta_{n_{k}+1}(\omega)\right) \\
&=l\left[d\left(\delta_{m_{k}}(\omega), \delta_{m_{k}+2}(\omega)\right)+d\left(\delta_{m_{k}+2}(\omega), \delta_{n_{k}+3}(\omega)\right)\right. \\
&\left.\quad+d\left(\delta_{n_{k}+3}(\omega), \delta_{n_{k}+1}(\omega)\right)\right] c\left[d\left(\eta_{m_{k}}(\omega), \eta_{m_{k}+2}(\omega)\right)\right. \\
&\left.\quad+d\left(\eta_{m_{k}+2}(\omega), \eta_{n_{k}+3}(\omega)\right)+d\left(\eta_{n_{k}+3}(\omega), \eta_{n_{k}+1}(\omega)\right)\right] \\
&=l\left[d\left(\delta_{m_{k}}(\omega), \delta_{m_{k}+2}(\omega)\right)\right. \\
& \quad+d\left(T\left(\omega,\left(\delta_{m_{k}+1}(\omega), \eta_{m_{k}+1}(\omega)\right)\right), S\left(\omega,\left(\delta_{n_{k}+2}(\omega), \eta_{n_{k}+2}(\omega)\right)\right)\right. \\
&\left.\quad+d\left(\delta_{n_{k}+3}(\omega), \delta_{n_{k}+1}(\omega)\right)\right]+c\left[d\left(\eta_{m_{k}}(\omega), \eta_{m_{k}+2}(\omega)\right)\right. \\
& \quad+d\left(T\left(\omega,\left(\eta_{m_{k}+1}(\omega), \delta_{m_{k}+1}(\omega)\right)\right), S\left(\omega,\left(\eta_{n_{k}+2}(\omega), \delta_{n_{k}+2}(\omega)\right)\right)\right. \\
&\left.\quad+d\left(\eta_{n_{k}+3}(\omega), \eta_{n_{k}+1}(\omega)\right)\right] \\
& \leq l\left[d\left(\delta_{m_{k}}(\omega), \delta_{m_{k}+2}(\omega)\right)+\left[l d\left(\delta_{n_{k}+2}(\omega), \delta_{m_{k}+1}(\omega)\right)\right.\right. \\
&\left.+c d\left(\eta_{n_{k}+2}(\omega), \eta_{m_{k}+1}(\omega)\right)+(1-(l+c)) d(A, B)\right] \\
&+d\left(\delta_{n_{k}+3}(\omega), \delta_{n_{k}+1}(\omega)\right)+c\left[d\left(\eta_{m_{k}}(\omega), \eta_{m_{k}+2}(\omega)\right)\right. \\
&+\left[l d\left(\eta_{n_{k}+2}(\omega), \eta_{m_{k}+1}(\omega)\right)+c d\left(\delta_{n_{k}+2}(\omega), \delta_{m_{k}+1}(\omega)\right)\right. \\
&\left.+(1-(l+c) d(A, B)]+d\left(\eta_{n_{k}+3}(\omega), \eta_{n_{k}+1}(\omega)\right)\right] \\
& \leq(l+c)\left[d\left(\delta_{m_{k}}(\omega), \delta_{m_{k}+2}(\omega)\right)+d\left(\delta_{n_{k}+3}(\omega), \delta_{n_{k}+1}(\omega)\right)\right. \\
&\left.+d\left(\eta_{m_{k}}(\omega), \eta_{m_{k}+2}(\omega)\right)+d\left(\eta_{n_{k}+3}(\omega), \eta_{n_{k}+1}(\omega)\right)\right] \\
&+(l+c)^{2}\left[d\left(\delta_{m_{k}+1}(\omega), \delta_{n_{k}+2}(\omega)\right)+d\left(\eta_{m_{k}+1}(\omega), \eta_{n_{k}+2}(\omega)\right)\right] \\
&+\left(1-(l+c)^{2}\right) d(A, B) .
\end{aligned}
$$

Letting $k \rightarrow \infty$, we get

$$
\begin{aligned}
d(A, B)+\epsilon^{\prime} & \leq(l+c)^{2}\left[d(A, B)+\epsilon^{\prime}\right]+\left(1-(l+c)^{2}\right) d(A, B) \\
& =d(A, B)+(l+c)^{2} \epsilon^{\prime}
\end{aligned}
$$

which contradicts. Therefore, we can concluded that (7) holds.

Step II. Now, we will show that $\left\{\delta_{n}(\omega)\right\},\left\{\eta_{n}(\omega)\right\},\left\{\delta_{n+1}(\omega)\right\}$ and $\left\{\eta_{n+1}(\omega)\right\}$ are Cauchy sequences. Since form $(3)$ and $(4)$, we have $d\left(\delta_{n}(\omega), \delta_{n+1}(\omega)\right) \rightarrow$ $d(A, B)$ and $d\left(\delta_{n+1}(\omega), \delta_{n+2}(\omega)\right) \rightarrow d(A, B)$. Since $(A, B)$ satisfies property $U C^{*}$, we get

$$
d\left(\delta_{n}(\omega), \delta_{n+2}(\omega)\right) \rightarrow 0 .
$$

Similarly, we also have $d\left(\delta_{n+1}(\omega), \delta_{n+3}(\omega)\right) \rightarrow 0$ because $(B, A)$ satisfies property $U C^{*}$. Here, we show that for every $\epsilon>0$ there exists an $N \in \mathbb{N}$ such that

$$
d\left(\delta_{m}(\omega), \delta_{n+1}(\omega)\right) \leq d(A, B)+\epsilon,
$$


for all $m \geq n \geq N$. Assume contrary, that there exists an $\epsilon>0$ such that for all $k \in \mathbb{N}$ there exists an $m_{k}>n_{k} \geq k$ such that

$$
d\left(\left(\delta_{m_{k}}(\omega),\left(\delta_{n_{k}+1}(\omega)\right) \leq d(A, B)+\epsilon .\right.\right.
$$

Now, we have

$$
\begin{aligned}
d(A, B)+\epsilon & <d\left(\delta_{m_{k}}(\omega), \delta_{n_{k}+1}(\omega)\right) \\
& \leq d\left(\delta_{m_{k}}(\omega), \delta_{n_{k}-1}(\omega)\right)+d\left(\delta_{n_{k}-1}(\omega), \delta_{n_{k}+1}(\omega)\right) \\
& \leq d(A, B)+\epsilon+d\left(\delta_{n_{k}-1}(\omega), \delta_{n_{k}+1}(\omega)\right)
\end{aligned}
$$

Letting $k \rightarrow \infty$, we have $d\left(\delta_{m_{k}}(\omega), \delta_{2 n_{k}+1}(\omega)\right) \rightarrow d(A, B)+\epsilon$. By using the triangle inequality and (7), we have

$$
\begin{aligned}
& d\left(\delta_{m_{k}}(\omega), \delta_{n_{k}+1}(\omega)\right. \\
& \leq d\left(\delta_{m_{k}}(\omega), \delta_{m_{k}+2}(\omega)+d\left(\delta_{m_{k}+2}(\omega), \delta_{n_{k}+3}(\omega)\right.\right. \\
& +d\left(\delta_{n_{k}+3}(\omega), \delta_{n_{k}+1}(\omega)\right. \\
& =d\left(\delta_{m_{k}}(\omega), \delta_{m_{k}+2}(\omega)+d\left(T\left(\omega,\left(\delta_{m_{k}+1}(\omega), \eta_{m_{k}+1}(\omega)\right)\right)\right. \text {, }\right. \\
& \left.S\left(\omega,\left(\delta_{n_{k}+2}(\omega), \eta_{n_{k}+2}(\omega)\right)\right)\right)+d\left(\delta_{n_{k}+3}(\omega), \delta_{n_{k}+1}(\omega)\right. \\
& \leq d\left(\delta_{m_{k}}(\omega), \delta_{m_{k}+2}(\omega)+\left[l d\left(\delta_{n_{k}+2}(\omega), \delta_{m_{k}+1}(\omega)\right)\right.\right. \\
& \left.+c d\left(\eta_{n_{k}+2}(\omega), \eta_{m_{k}+1}(\omega)\right)+(1-(l-c)) d(A, B)\right] \\
& +d\left(\delta_{n_{k}+3}(\omega), \delta_{n_{k}+1}(\omega)\right. \\
& =l\left[d\left(T\left(\omega,\left(\delta_{n_{k}+1}(\omega), \eta_{n_{k}+1}(\omega)\right)\right), S\left(\omega,\left(\delta_{m_{k}}(\omega), \eta_{m_{k}}(\omega)\right)\right)\right)\right] \\
& +c\left[d\left(T\left(\omega,\left(\eta_{n_{k}+1}(\omega), \delta_{n_{k}+1}(\omega)\right)\right), S\left(\omega,\left(\eta_{m_{k}}(\omega), \delta_{m_{k}}(\omega)\right)\right)\right)\right] \\
& +(1-(l-c)) d(A, B)+d\left(\delta_{m_{k}}(\omega), \delta_{m_{k}+2}(\omega)\right. \\
& +d\left(\delta_{n_{k}+3}(\omega), \delta_{n_{k}+1}(\omega)\right. \\
& \leq l\left[l d\left(\delta_{m_{k}}(\omega), \delta_{n_{k}+1}(\omega)\right)+c d\left(\eta_{m_{k}}(\omega), \eta_{n_{k}+1}(\omega)\right)\right. \\
& +(1-(l-c)) d(A, B)]+\left[l d\left(\eta_{m_{k}}(\omega), \eta_{n_{k}+1}(\omega)\right)\right. \\
& \left.+c d\left(\delta_{m_{k}}(\omega), \delta_{n_{k}+1}(\omega)\right)+(1-(l-c)) d(A, B)\right] \\
& +(1-(l-c)) d(A, B)+d\left(\delta_{m_{k}}(\omega), \delta_{m_{k}+2}(\omega)\right. \\
& +d\left(\delta_{n_{k}+3}(\omega), \delta_{n_{k}+1}(\omega)\right. \\
& \leq(l+c)^{2}\left[d\left(\delta_{m_{k}}(\omega), \delta_{n_{k}+1}(\omega)\right)+d\left(\eta_{m_{k}}(\omega), \eta_{n_{k}+1}(\omega)\right)\right] \\
& +\left(1-(l-c)^{2}\right) d(A, B)+d\left(\delta_{m_{k}}(\omega), \delta_{m_{k}+2}(\omega)\right. \\
& +d\left(\delta_{n_{k}+3}(\omega), \delta_{n_{k}+1}(\omega)\right. \\
& <(l+c)^{2}(d(A, B)+\epsilon)+\left(1-(l-c)^{2}\right) d(A, B) \\
& +d\left(\delta_{m_{k}}(\omega), \delta_{m_{k}+2}(\omega)+d\left(\delta_{n_{k}+3}(\omega), \delta_{n_{k}+1}(\omega)\right.\right. \\
& =(l+c)^{2} \epsilon+d(A, B)+d\left(\delta_{m_{k}}(\omega), \delta_{m_{k}+2}(\omega)\right. \\
& +d\left(\delta_{n_{k}+3}(\omega), \delta_{n_{k}+1}(\omega)\right. \text {. }
\end{aligned}
$$


Letting $k \rightarrow \infty$, we get

$$
d(A, B)+\epsilon \leq d(A, B)+(l+c)^{2} \epsilon,
$$

a contradiction, because $l+c<1$. Therefore, condition (9) holds. By (9) and

$$
d\left(\delta_{n}(\omega), \delta_{n+1}(\omega)\right) \rightarrow d(A, B)
$$

and using the property $U C^{*}$ of $(A, B)$, we have that $\delta_{n}(\omega)$ is a Cauchy sequences. In a similar way, we can prove that $\left\{\eta_{n}(\omega)\right\},\left\{\delta_{n+1}(\omega)\right\}$ and $\left\{\eta_{n+1}(\omega)\right\}$ are Cauchy sequences.

Step III. Since $A$ and $B$ are subsets of a complete separable metric space $X$. Therefore there exists $\delta(\omega)$ and $\eta(\omega)$ such that $\delta_{n}(\omega) \rightarrow \delta(\omega)$ and $\eta_{n}(\omega) \rightarrow \eta(\omega)$. we have

$$
\begin{aligned}
d(A, B) & \leq d\left(\delta(\omega), \delta_{n-1}(\omega)\right) \\
& \leq d\left(\delta(\omega), \delta_{n}(\omega)\right)+d\left(\delta_{n}(\omega), \delta_{n-1}(\omega)\right) .
\end{aligned}
$$

Letting $n \rightarrow \infty$, we get $d\left(\delta(\omega), \delta_{n-1}(\omega)\right) \rightarrow d(A, B)$. By a similar argument, we can also get $d\left(\eta(\omega), \eta_{n-1}(\omega)\right) \rightarrow d(A, B)$. It follows that

$$
\begin{aligned}
& d\left(\delta_{n}(\omega), S(\omega,(\delta(\omega), \eta(\omega)))\right) \\
&= d\left(T\left(\omega,\left(\delta_{n-1}(\omega), \eta_{n-1}(\omega)\right)\right), S(\omega,(\delta(\omega), \eta(\omega)))\right) \\
& \leq {\left[l d\left(\delta(\omega), \delta_{n-1}(\omega)\right)+c d\left(\eta(\omega), \eta_{n-1}(\omega)\right)\right] } \\
&+(1-(l+c)) d(A, B) .
\end{aligned}
$$

Letting $n \rightarrow \infty$, we get

$$
d(\delta(\omega), S(\omega,(\delta(\omega), \eta(\omega))))=d(A, B) .
$$

Similarly, we can prove that

$$
d(\eta(\omega), S(\omega,(\eta(\omega), \delta(\omega))))=d(A, B) .
$$

Therefore, we have $(\delta(\omega), \eta(\omega))$ is a random coupled best proximity point of $S$. By the same argument, we can prove that there exist $\delta^{\prime}(\omega), \eta^{\prime}(\omega) \in B$ such that $\delta_{n+1}(\omega) \rightarrow \delta^{\prime}(\omega)$ and $\eta_{n+1}(\omega) \rightarrow \eta^{\prime}(\omega)$. Moreover, we also have

$$
d\left(\delta^{\prime}(\omega), T\left(\omega,\left(\delta^{\prime}(\omega), \eta^{\prime}(\omega)\right)\right)\right)=d(A, B),
$$

and

$$
d\left(\eta^{\prime}(\omega), T\left(\omega,\left(\eta^{\prime}(\omega), \delta^{\prime}(\omega)\right)\right)\right)=d(A, B) .
$$

and so $\left(\delta^{\prime}(\omega), \eta^{\prime}(\omega)\right)$ is a coupled random best proximity point of $T$.

Here, we note that if $(A, B)$ is a pair of nonempty closed subsets of a uniformly convex Banach space $X$ such that $A$ is convex, then $(A, B)$ has the property $U C^{*}$. Then, we have the following corollary. 
Corollary 1. Given $(X, d)$ be a Polish space and $(\Omega, \Sigma)$ be a measurable space. Given $A, B$ be nonempty closed subsets of a uniformly convex separable Banach space $X$. Suppose $S: \Omega \times(A \times A) \rightarrow B$ is a operator and $T: \Omega \times(B \times B) \rightarrow A$ is a random operator. Define

$$
x_{n+1}(\omega)=S\left(\omega, x_{n}(\omega), y_{n}(\omega)\right), y_{n+1}(\omega)=S\left(\omega, y_{n}(\omega), x_{n}(\omega)\right),
$$

and

$$
x_{n+2}(\omega)=T\left(\omega, x_{n+1}(\omega), y_{n+1}(\omega)\right), y_{n+2}(\omega)=T\left(\omega, y_{n+1}(\omega), x_{n+1}(\omega)\right),
$$

for all $n \in \mathbb{N}$ and $\omega \in \Omega$. Given $S$ is continuous and suppose that

(a) $S(\cdot, v)$ is measurable for all $v \in A \times A$ and $T(\cdot, u)$ is measurable for all $u \in B \times B$.

(b) $(S, T)$ is a generalized $\omega$-cyclic contraction.

Then $S$ and $T$ have a random coupled best proximity point.

Next, we give some illustrative example of Corollary 1.

Example 2. Cosider a uniformly convex separable Banach space $X=\mathbb{R}$ with the usual norm. Let $\Omega=[0,1]$ and let $\sigma$ be the sigma algebra of Lebesgues measurable subset of $[0,1]$. Let $A=[1,2]$ and $B=[-2,-1]$. It easy to see that $d(A, B)=2$. Define random operators $S: \Omega \times(A \times A) \rightarrow B$ and $T: \Omega \times(B \times B) \rightarrow A$ by

$$
S\left(\omega,\left(a(\omega), a^{\prime}(\omega)\right)\right)=\frac{-2 a(\omega)-3 a^{\prime}(\omega)-1}{6},
$$

and

$$
T\left(\omega,\left(b(\omega), b^{\prime}(\omega)\right)\right)=\frac{-2 b(\omega)-3 b^{\prime}(\omega)+1}{6} .
$$

For arbitrary $\left(a(\omega), a^{\prime}(\omega)\right) \in A \times A$ and $\left(b(\omega), b^{\prime}(\omega)\right) \in B \times B$ and fixed $l=\frac{1}{2}$ and $c=\frac{1}{3}$, we get

$$
\begin{aligned}
d & \left.S\left(\omega,\left(a(\omega), a^{\prime}(\omega)\right)\right), T\left(\omega,\left(b(\omega), b^{\prime}(\omega)\right)\right)\right) \\
& =\left|\frac{-2 a(\omega)-3 a^{\prime}(\omega)-1}{6}-\frac{-2 b(\omega)-3 b^{\prime}(\omega)+1}{6}\right| \\
& \leq \frac{2|a(\omega)-b(\omega)|+3\left|a^{\prime}(\omega)-b^{\prime}(\omega)\right|}{6}+\frac{1}{3} \\
& =\left[l d(a(\omega), b(\omega))+c d\left(a^{\prime}(\omega), b^{\prime}(\omega)\right)\right]+(1-(l+c) d(A, B) .
\end{aligned}
$$

This implies that $(S, T)$ is a generalized $\omega$-cyclic contraction with $l=\frac{1}{2}$ and $c=\frac{1}{3}$. Since $A$ and $B$ are convex, we have $(A, B)$ and $(B, A)$ satisfy the property $U C^{*}$. Therefore, all hypothesis of Corollary 1 hold. So $S$ and $T$ have a random coupled best proximity point. We note that a point $(1,1) \in A \times A$ is a unique random coupled best proximity point of $S$ and $(-1,-1) \in B \times B$ is a unique random coupled best proximity point of $T$. 
Theorem 2. Given $(X, d)$ be a Polish space and $(\Omega, \Sigma)$ be a measurable space. Given $A, B$ be nonempty compact subsets of $X$. Suppose $S: \Omega \times$ $(A \times A) \rightarrow B$ is a random operator and $T: \Omega \times(B \times B) \rightarrow A$ is a random operator. Define

$$
x_{n+1}(\omega)=S\left(\omega,\left(x_{n}(\omega), y_{n}(\omega)\right)\right), y_{n+1}(\omega)=S\left(\omega,\left(y_{n}(\omega), x_{n}(\omega)\right)\right),
$$

and

$$
x_{n+2}(\omega)=T\left(\omega,\left(x_{n+1}(\omega), y_{n+1}(\omega)\right)\right), y_{n+2}(\omega)=T\left(\omega,\left(y_{n+1}(\omega), x_{n+1}(\omega)\right)\right),
$$

for all $n \in \mathbb{N}$ and $\omega \in \Omega$. Given $S$ is continuous and suppose that

(a) $S(\cdot, v)$ is measurable for all $v \in A \times A$ and $T(\cdot, u)$ is measurable for all and $u \in B \times B$.

(b) $(A, B)$ and $(B, A)$ have the property $U C^{*}$.

(c) $(S, T)$ is a generalized $\omega$-cyclic contraction.

Then $S$ and $T$ have a random coupled best proximity point.

Proof. By the same argument of the proof of Theorem 2, we have $\delta: \Omega \rightarrow X$ is a measurable mapping and $\eta: \Omega \rightarrow X$ is a measurable mapping. We have

$$
\begin{gathered}
\delta_{n+1}(\omega)=S\left(\omega,\left(\delta_{n}(\omega), \eta_{n}(\omega)\right)\right), \eta_{n+1}(\omega)=S\left(\omega,\left(\eta_{n}(\omega), \delta_{n}(\omega)\right)\right), \\
\delta_{n+2}(\omega)=T\left(\omega,\left(\delta_{n+1}(\omega), \eta_{n+1}(\omega)\right)\right), \eta_{n+2}(\omega)=T\left(\omega,\left(\eta_{n+1}(\omega), \delta_{n+1}(\omega)\right)\right),
\end{gathered}
$$

for all $n \in \mathbb{N} \cup\{0\}$. We have $\delta_{n}(\omega), \eta_{n}(\omega) \in A$ and $\delta_{n+1}(\omega), \eta_{n+1}(\omega) \in B$ for all $n \in \mathbb{N} \cup\{0\}$. Since $A$ is a compact. The sequences $\left\{\delta_{n}(\omega)\right\}$ has convergent subsequences $\left\{\delta_{n_{k}}(\omega)\right\}$ and he sequences $\left\{\eta_{n}(\omega)\right\}$ has convergent subsequences $\left\{\eta_{n_{k}}(\omega)\right\}$. That is, $\delta_{n_{k}}(\omega) \rightarrow \delta(\omega)$ and $\eta_{n_{k}}(\omega) \rightarrow \eta(\omega)$. Now, we have

$$
\begin{aligned}
d(A, B) & \leq d\left(\delta(\omega), \delta_{n_{k}-1}(\omega)\right) \\
& \leq d\left(\delta(\omega), \delta_{n_{k}}(\omega)\right)+d\left(\delta_{n_{k}}(\omega), \delta_{n_{k}-1}(\omega)\right) .
\end{aligned}
$$

By (3), we have $d\left(\delta_{n_{k}}(\omega), \delta_{n_{k}-1}(\omega)\right) \rightarrow d(A, B)$. Letting $k \rightarrow \infty$ in (10), we get

$$
d\left(\delta(\omega), \delta_{n_{k}-1}(\omega)\right) \rightarrow d(A, B) .
$$

Similar argument show that

$$
d\left(\eta(\omega), \delta_{n_{k}-1}(\omega)\right) \rightarrow d(A, B)
$$

Note that

$$
\begin{aligned}
d(A, B) \leq & d\left(\delta_{n_{k}}(\omega), S(\omega,(\delta(\omega), \eta(\omega)))\right) \\
= & d\left(T\left(\omega,\left(\delta_{n_{k}-1}(\omega), \eta_{n_{k}-1}(\omega)\right)\right), S(\omega,(\delta(\omega), \eta(\omega)))\right) \\
\leq & {\left[l d\left(\delta(\omega), \delta_{n_{k}-1}(\omega)\right)+c d\left(\eta(\omega), \eta_{n_{k}-1}(\omega)\right)\right.} \\
& +(1-(l+c)) d(A, B)]
\end{aligned}
$$


Letting $k \rightarrow \infty$, we get $d(\delta(\omega), S(\omega,(\delta(\omega), \eta(\omega))))=d(A, B)$. Similarly, it can be proved that

$$
d(\eta(\omega), S(\omega,(\eta(\omega), \delta(\omega))))=d(A, B) .
$$

Therefore $S$ has a random coupled best proximity point $(\delta(\omega), \eta(\omega))$. Similar, since $B$ is compact, it can be proved that $T$ has a random coupled best proximity point.

Acknowledgements. The first author thanks to the Petchra Pra Jom Klao Doctoral Scholarship for Ph.D. program at KMUTT. This project was supported by the Theoretical and Computational Science (TaCS) Center under Computational and Applied Science for Smart Innovation Cluster (CLASSIC), Faculty of Science, KMUTT.

\section{References}

[1] Akbar F., Kutbi M.A., Shah M.H., Shafqat N., Random coupled and tripled best proximity results with cyclic contraction in metric spaces, J. Nonlinear Sci. Appl., 9(3)(2016), 940-956.

[2] Anh T.N., Random equations and applications to general random fixed point theorems, New Zealand J. Math., 41(2011), 17-24.

[3] BEg I., Approximation of random fixed points in normed spaces, Nonlinear Anal., 51(8)(2002), 1363-1372.

[4] Beg I., Shahzad N., An application of a random fixed point theorem to random best approximation, Arch. Math., (Basel), 74(4)(2000), 298-301.

[5] Bharucha-Reid A.T., Random integral equations, Academic Press, New York-London, Mathematics in Science and Engineering, 96(1972).

[6] Bharucha-Reid A.T., Fixed point theorems in probabilistic analysis, Bull. Amer. Math. Soc., 82(5)(1976), 641-657.

[7] Choudhury B.S., Convergence of a random iteration scheme to a random fixed point, J. Appl. Math. Stochastic Anal., 8(2)(1995), 139-142.

[8] Ćirić L. Lakshmikantham V., Coupled random fixed point theorems for nonlinear contractions in partially ordered metric spaces, Stoch. Anal. Appl., $27(6)(2009), 1246-1259$.

[9] Di Bari C, Suzuki T., Vetro C., Best proximity points for cyclic Meir-Keeler contractions, Nonlinear Anal., 9(11)(2008), 3790-3794.

[10] Fan K., Extensions of two fixed point theorems of F. E. Browder, Math. Z., 112(1969), 234-240.

[11] Gupta A., Rajput S.S., Kaurav P.S., Coupled Best Proximity Point Theorem in Metric Spaces, International Journal of Analysis and Applications, $4(2)(2014), 201-215$.

[12] Hanš O., Reduzierende zufällige Transformationen, Czechoslovak Math. J., 7(82)(1957), 154-158.

[13] Hanš O., Random operator equations, in Proc. 4th Berkeley Sympos. Math. Statist. and Prob., Univ. California Press, Berkeley, Calif., Vol. II, (1961), 185-202. 
[14] Hussain N., Latif A., Shafqat N., Weak contractive inequalities and compatible mixed monotone random operators in ordered metric spaces, $J$. Inequal. Appl., 20(2012), 257.

[15] Ітон S., A random fixed point theorem for a multivalued contraction mapping, Pacific J. Math., 68(1)(1977), 85-90.

[16] Khan A.R., Hussain N., Yasmin N., Shafqat N., Random coincidence point results for weakly increasing functions in partially ordered metric spaces, Bull. Iranian Math. Soc., 41(2)(2015), 407-422.

[17] Kumam P., Fixed point theorem and random fixed point theorem for set-valued non-self mappings, Thai J. Math., 2(2)(2004), 295-307.

[18] Kumam P., Random common fixed points of single-valued and multivalued random operators in a uniformly convex Banach space, J. Comput. Anal. Appl., 13(2)(2011), 368-375.

[19] Kumam P., Plubtieng S., Some random fixed point theorems for non-self nonexpansive random operators, Turkish J. Math., 30(4)(2006), 359-372.

[20] Kumam P. Plubtieng S., The characteristic of noncompact convexity and random fixed point theorem for set-valued operators, Czechoslovak Math. J., 57(132)(1)(2007), 269-279.

[21] Kumam W., Kumam P., Some random fixed point theorems for random asymptotically regular operators, Demonstratio Math., 42(1)(2009), 131-141.

[22] Kumam W., Kumam P., Random fixed points of multivalued random operators with property (D), Random Oper. Stoch. Equ., 15(2)(2007), 127-136.

[23] Kumam W., Kumam P., Random fixed point theorems for multivalued subsequentially limit-contractive maps satisfying inwardness conditions, J. Comput. Anal. Appl., 14(2)(2012), 239-251.

[24] Mongkolkeha C., Cho Y.J.,Kumam P., Best proximity points for generalized proximal $C$-contraction mappings in metric spaces with partial orders, J. Inequal. Appl., 94(2013), pages 12.

[25] Mongkolkeha C., Cho Y.J .J., Kumam P., Best proximity points for Geraghty's proximal contraction mappings, Fixed Point Theory Appl., 180(2013), pages 17 .

[26] Mongkolkeha C., Kumam P., Best proximity point theorems for generalized cyclic contractions in ordered metric spaces, J. Optim. Theory Appl., $155(1)(2012), 215-226$.

[27] Nashine H.K., Kumam P., Vetro C., Best proximity point theorems for rational proximal contractions, Fixed Point Theory Appl., 95(2013), pages 11.

[28] Rockafellar, R.T., Measurable dependence of convex sets and functions on parameters, J. Math. Anal. Appl., 28(1969), 4-25.

[29] Sanhan W., Mongkolkeha C., Kumam P., Generalized proximal $\psi$-contraction mappings and best proximity points, Abstr. Appl. Anal., (2012), Art. ID 896912, pages 19.

[30] Sintunavarat W., Kumam P., Coupled best proximity point theorem in metric spaces, Fixed Point Theory Appl., 93(2012), pages 16.

[31] Suzuki T., Kikkawa M., Vetro C., The existence of best proximity points in metric spaces with the property UC, Nonlinear Anal., 71(7-8)(2009), 2918-2926. 
[32] Vetro C., Best proximity points: convergence and existence theorems for p-cyclic mappings, Nonlinear Anal., 73(7)(2010), 2283-2291.

[33] ŠPAČEK A., Zufällige Gleichungen, Czechoslovak Mathematical Journal, $5(4)(1955), 462-466$.

[34] Wagner D.H., Survey of measurable selection theorems: an update. In Measure theory, Oberwolfach 1979 (Proc. Conf., Oberwolfach, 1979), vol. 794 of Lecture Notes in Math., pages 176-219, Springer, Berlin-New York, 1980.

[35] Zhu X.-H., XiaO J.-Z., Random periodic point and fixed point results for random monotone mappings in ordered Polish spaces, Fixed Point Theory Appl., (2010), Art. ID 723216, pages 13.

\author{
POOM KUMAM \\ Department of Mathematics \\ FACULTY OF SCIENCE \\ King Mongkuts University of Technology Thonburi (KMUTT) \\ BANGKOK 10140, THAILAND \\ e-mail: poom.kum@kmutt.ac.th \\ Chayut Kongban \\ Department of Mathematics \\ FACULTY OF SCIENCE \\ King Mongkuts University of Technology Thonburi (KMUTT) \\ BANGKOK 10140, THAILAND \\ e-mail: chayut_kb@hotmail.com
}

Received on 19.01.2017 and, in revised form, on 14.09.2017. 